\title{
Las comunidades afrocolombianas y su realidad en el Estado Social de Derecho: ¿Hacia su reconocimiento jurídico en un mundo de discriminación social? Casos Neiva, Baraya y La Plata: aspectos laborales, afectivos y educativos
}

Semillero de Investigación Cimarrón

Edinson Quintero Zambrano

Germán David Quintero Castro

Luis Guillermo Cardozo

Tutora - Doctora Martha Cecilia Abella de Fierro

Línea de Investigación Nuevos Paradigmas del Derecho

Grupo de Investigación Conciencia Jurídica / Universidad Surcolombiana

\section{RESUMEN}

Con la Constitución Política de 1991, el pueblo Afrocolombiano renace y adquiere la relevancia que se merece en un Estado Social de Derecho. La diversidad étnica y cultural de la Nación es reconocida como principio fundamental y así lo registran los artículos 7, 8, 9, 68, 70 y 72 .

Corresponde identificar si la sociedad se ha apropiado de estas posturas jurídicas permitiendo a los afrocolombianos hacer parte de su entorno no solo desde lo constitucional sino desde lo cotidiano. Con este texto se busca evaluar si aquel discurso, ha tenido o no aplicabilidad en los Municipios de Neiva, Baraya y La Plata - Huila - en lo concerniente a la vida laboral, afectiva y educativa de estos grupos. 


\section{PALABRAS CLAVE}

Afrocolombiano, Etnia, Cultura, Estado Social de Derecho, Democracia, Discriminación, Derecho al Trabajo, Derecho a la Educación, Derecho a la Consulta Previa, Afectividad.

\section{ABSTRACT}

Along with the promulgation of the Political Constitution of 1991 the Afrocolombian people are, in line with the so-called "Social State of Law", rescued from a historical normative ostracism. Cultural and ethnic diversity vindicates its character as a fundamental principle of law. The Colombian Chart Magna refers to our cultural patrimony in more that few articles, among which No. 7, 8, 9, 68, 70 y 72 are worth to mention.

The analysis of the actual social positioning of Afrocolombian communities from different perspectives, i.e., educational and labor conditions, daily economic standards, or even emotional interactions, will provide for broader basis in order to understand whether the constitutional discourse has been effectuated in practice. This paper pursues the aforementioned analysis for a geographically limited scope, namely the municipalities of Neiva, Baraya and La Plata, all located in the Department of Huila.

\section{KEYWORDS}

Afrocolombian, Ethnic, Culture, Social State of Law, Democracy, Discrimination, Right to Work, Right to Education, Right to Previous Consultation, Affectivity.

\section{INTRODUCCIÓN}

El Estado colombiano tiene importantes fortalezas jurídicas en el área de los Derechos Fundamentales y en el tema de los Derechos Humanos, lo cual se ve reflejado en su normatividad, jurisprudencia y doctrina bastante garantistas. Importantes instituciones como la Corte Constitucional y la Defensoría del Pueblo, se destacan por la defensa de la persona y la dignidad humana. Igualmente, ha de reconocerse que la Rama Judicial ha tomado un protagonismo 
relevante al intervenir y resolver las diferentes expresiones de violación y desconocimiento de los Derechos Fundamentales, Económicos, Sociales y Culturales, Colectivos y Ambientales mediante las diferentes acciones de amparo donde se destacan las Acciones de Tutela, de Cumplimiento, Populares y de Grupo, al igual que el Habeas Corpus, el Habeas Data y el mismo Derecho de Petición.

Sin embargo, las Entidades Públicas, del orden nacional y territorial no han logrado adecuar sus programas y proyectos en el marco de unas políticas públicas con énfasis en Derechos Humanos. Esto significa que aún nos falta mucho camino por recorrer para lograr consolidar una nueva visión del Estado: Un Estado Social de Derecho sustentado en los principios de la Dignidad Humana, la Solidaridad y el Interés General. No es suficiente obtener y desarrollar avances teóricos jurídico - políticos si no hay prácticas sociales y compromisos estatales que conduzcan hacia una mejor calidad de vida para todos los colombianos y colombianas.

Son muchos los debates y discursos jurídicos y académicos que pregonan por la defensa y reconocimiento de los Derechos Humanos, el respeto a la diferencia y el rechazo a prácticas discriminatorias o excluyentes con ocasión al pensamiento, la expresión, el credo o la raza, pero ¿la realidad cotidiana refleja estas posturas intelectuales? ¿Realmente el Estado ha implementado políticas dirigidas a garantizar el pleno disfrute de los Derechos Humanos?

Siendo la Dignidad Humana y la Solidaridad los pilares de los Derechos Humanos no es entendible cómo se detectan posturas discriminatorias, especialmente en relación con los grupos étnicos: Indígenas y Afrocolombianos. Esta discriminación es de carácter indirecta, por cuanto "no tiene como soporte una intencionalidad individual o colectiva explícita (por ejemplo, jurídico-institucional), sino que su sostén son las "costumbres" y "tradiciones", conceptualización de gran utilidad para analizar el fenómeno de los países latinoamericanos. En otras palabras, se trata de un imaginario colectivo que opera inconscientemente y afecta los distintos espacios de la vida cotidiana y pública, no obstante el enunciado del principio 
universal de igualdad de tratamiento y oportunidades, que ha sido consignado en la mayor parte de las constituciones republicanas de estos países a lo largo de los siglos XIX y XX" (Urrea, y Barbary, 2004)

La discriminación es un acto de rechazo hacia las comunidades AFROCOLOMBIANAS cuyo prefijo hace referencia a la herencia cultural africana que pervive en la memoria colectiva a través de distintos saberes y prácticas, sobre todo en la tradición oral, la memoria corporal, que incluyen gestos, la danza, las palabras, el arte, la música, los cantos y su relación con la naturaleza. Es el pasado ancestral, elemento clave de su identidad étnica hoy, o de color, violando muchos de sus derechos fundamentales como el Derecho a la Igualdad, Trabajo, Familia, Educación, e incluso, a tener una Familia y tener la opción libre de formar pareja sin sentir rechazo social alguno. La Ley 70 de 1993 reconoce importantes Derechos a favor de las etnias negras entre los que se destacan la Educación y Propiedad de tierras. Sin embargo, en la cotidianidad se presentan grandes inconvenientes debido, no solo, al total desconocimiento de dichas normas, sino además, por los continuos atentados contra la dignidad humana y la solidaridad, desde la discriminación en un establecimiento educativo hasta el hecho de conformar una familia.

A partir de la Constitución Política de 1991, como resultado de un proceso histórico - jurídico en materia de Derechos Humanos, el Estado colombiano reconoce de manera enfática los Derechos Fundamentales de todos los habitantes de la nación con el fin de lograr un equilibrio de intereses colectivos y de asegurar su interrelación en términos de igualdad. De manera especial realizó el reconocimiento de los derechos propios de las COMUNIDADES AFROCOLOMBIANAS, estableciendo así un tema novedoso para las instituciones y la población en general; pero no ha sido fácil la implementación de estas nuevas formas de vida donde los Derechos Humanos logren la dignificación de estos seres humanos. Existe aún desconocimiento sobre sus derechos, su historia y dinámicas culturales, sus problemas y sus conflictos de supervivencia. 
Aunque resulta paradójico, el pueblo afrocolombiano no contó con un representante en la Asamblea Nacional Constituyente. A pesar de lo anterior, el movimiento afrocolombiano mediante la presión y la "representación indirecta", logra la aprobación del artículo transitorio 55. Este logro fue significativo y decisivo debido a que "en primer lugar, por primera vez en una Constitución colombiana y en toda la legislación republicana se introduce la categoría comunidades negras. Con el uso de esta categoría, los negros, que habían sido invisibilizados (Friedemann, 1984) por el proyecto de nación mestiza, se hacen visibles. Son reconocidos como un grupo étnico cuya identidad se basa en el desarrollo de unas prácticas tradicionales de producción y en la ocupación ancestral de las riberas de los grandes ríos de la Costa Pacífica. En segundo lugar, el Estado, además de reconocer esta identidad cultural, adquiere el compromiso constitucional de protegerla $y$ fomentarla. En tercer lugar, se reconoce el derecho a la propiedad colectiva sobre las tierras ancestralmente ocupadas por los negros en la Costa Pacífica colombiana, la zona ampliamente rica en biodiversidad en la que los macro proyectos desarrollistas del capitalismo global estaba poniendo en peligro los territorios construidos por la gente negra" (Castillo, 2006).

Además, al interior de las comunidades AFROCOLOMBIANAS existen serios problemas que agravan su situación, tales como la carencia de territorios propios, el bajo nivel de organización, la pérdida parcial de elementos de identidad y el desconocimiento de sus Derechos Constitucionales (C. P. 1991) y de sus garantías reconocidas no solo por la ley 70 de 1993, sino en otras disposiciones normativas internas como la Ley 115 de 1.994, Ley 725 de 2.001, Ley 649 de 2.001, Decreto 804 de 1995 y Decreto 1122 de 1.998 e internacionales como la Convención Internacional sobre la Eliminación de todas las Formas de Discriminación Racial, que les identifica como etnias que merecen protección y respeto. La situación se agrava con el creciente marginamiento de sus sistemas productivos, en tanto que no cuentan con proyectos que garanticen la supervivencia adecuada a una buena calidad de vida. 
En ese orden de ideas, a continuación mostraremos los resultados de nuestro trabajo investigativo, dando a conocer la cruda realidad que aún viven las comunidades Afrocolombianas de los Municipios de Neiva, Baraya y La Plata.

\section{OBJETIVOS}

\section{General}

Identificar y analizar la situación de las comunidades AFROCOLOMBIANAS del Departamento del Huila, en los Municipios de Neiva, Baraya y La Plata y su realidad en el Estado Social de Derecho y determinar cuál ha sido su reconocimiento familiar, laboral y social en un mundo paradójico de discriminación y exclusión pero con un importante desarrollo jurídico.

\section{Específicos}

- Evaluar la situación afectiva, familiar, laboral y educativa de las comunidades Afrocolombianas residenciadas en los municipios de Neiva, Baraya y la Plata, en el Departamento del Huila.

- Determinar la aceptación o rechazo social de los Afrocolombianos en los municipios de Neiva, Baraya y la Plata, estudiando estadísticamente los niveles de discriminación y exclusión.

- Determinar las causas por las cuales las comunidades Afrodescendientes carecen de visibilidad ante la sociedad huilense.

- Estudiar la situación de la conformación de familia en lo pluriétnico y las relaciones afectivas de las mismas.

\section{METODOLOGÍA}

El objetivo de la investigación descriptiva consiste en llegar a conocer las situaciones, costumbres y actitudes predominantes a través de la descripción exacta de las actividades, objetos, procesos y personas. Su meta no se limita a la 
recolección de datos, sino a la predicción e identificación de las relaciones que existen entre dos o más variables. Los investigadores no son meros tabuladores, sino que recogen los datos sobre la base de una hipótesis o teoría, exponen y resumen la información de manera cuidadosa y luego analizan minuciosamente los resultados, a fin de extraer generalizaciones significativas que contribuyan al conocimiento.

Teniendo en cuenta la problemática a abordar, se considera que el enfoque epistemológico más conveniente para desarrollar la presente investigación es el de la investigación cuantitativa descriptiva, puesto que comprenderá la descripción, registro, análisis, diagnostico e interpretación de la situación actual de las comunidades Afrocolombianas en el departamento del Huila (Neiva, la Plata y Baraya).

\section{POBLACIÓN}

El muestreo fue tomado tanto de personas negras como blancas de los municipios de Neiva, la Plata y Baraya del Departamento del Huila.

\section{MUESTRA}

Teniendo en cuenta que la población de Afrocolombianos involucrada en el problema es bastante amplia, se hizo necesario tomar una muestra representativa de esta. En consecuencia, la investigación se hizo sobre un 30\% de la población mencionada.

\section{RECOLECCIÓN DE DATOS}

La investigación se realizó mediante las siguientes actividades:

- Aplicación de encuestas en las zonas establecidas para la investigación. 
- Identificación de la situación mediante un diálogo directo con visitas a las comunidades Afrocolombianas de Neiva, la Plata y Baraya, (familias, organizaciones y asociaciones).

- Realizar un análisis y estudio de la jurisprudencia constitucional referente a estos regímenes especiales.

\section{ELABORACIÓN DE FICHAS DE TRABAJO}

Se aplicó una ficha de trabajo con la cual se pretendió despejar dudas sobre la situación actual de las comunidades Afrocolombianas residentes en Neiva, la Plata y Baraya.

\section{PROCESAMIENTO DE DATOS Y CODIFICACIÓN}

Una vez recopilados los datos mediante los instrumentos diseñados para tal fin, se procesaron y sistematizaron, para cuantificarlos matemáticamente, lo cual permitió aplicar formulas estadísticas y efectuar posteriormente, el análisis de los resultados y llegar a las conclusiones en relación con la problemática planteada.

\section{DESARROLLO}

\section{ANÁLISIS DE LA SITUACIÓN DE LOS AFROCOLOMBIANOS ANTE ALGUNOS DERECHOS FUNDAMENTALES}

\section{Derecho al trabajo}

¿Cuál es la realidad actual del derecho al trabajo de la población afro descendiente en el país?

La problemática social y económica que afronta el país es de gran magnitud y debe tenerse en cuenta a la hora de establecer la realidad de determinada población y en este caso particular, del estado de la población afro frente al derecho constitucional al trabajo. Es preocupante ver como en las diferentes esferas de la relación social, permanecen aún patrones contribuyentes a la 
violación de derechos inherentes de las personas, partiendo de supuestos tales como la discriminación por razones de raza, color, sexo, creencias, etc.

La realidad colombiana en torno a la situación laboral de la población afro es alarmante, pues está calculado que de los 43.7 millones de habitantes cerca de los 3.448.389 habitantes que representa este grupo étnico, o sea, el $8 \%$ del total de la población del país vive en condiciones supremamente denigrantes, en situación de abandono por parte de la sociedad y del Estado mismo, pues el 14\% de la población afro colombiana se encuentra en condiciones de desempleo.

Para el año de 2010, se estableció que el desempleo alcanzó el $14.8 \%$ de la población total del país es decir 6.660 .000 personas desempleadas (Departamento Administrativo Nacional de Estadística, 2010); sin embargo, ello no significa que el resto de la población colombiana se encuentre laborando en condiciones dignas y en empleos estables, pues son muchas las personas ubicadas en empleos temporales o simplemente empleos como el famoso "rebusque" de gran relevancia en las familias colombianas y en especial de las familias afro descendientes que hoy por hoy son una de las poblaciones más golpeadas frente al fenómeno de la desocupación. Por otro lado vale la pena mencionar, además de lo anterior, que a la población afro en nuestro territorio se le reconoce por los oficios de alto riesgo que desempeña, sin las condiciones mínimas de seguridad y salubridad, en minas y canteras.

En la actualidad es notorio como ya se había indicado, que la población en general se esté exponiendo a la práctica de actividades donde se desconocen las garantías y los derechos mínimos establecidos en la constitución y la ley para con los trabajadores. La población afrocolombiana, además de ser una de las poblaciones con más altos índices de desempleo, es además la población mas "utilizada" para efectos de empleo, en trabajos o actividades de gran peligrosidad. Actividades como la minería, en donde la población afro tiene gran acogida laboral, dado su gran nivel de resistencia física, entre otros factores, es una de las más proclive a afectar los derechos de aquellas personas que laboran en la explotación de minas cualquiera que sea su denominación, pues las condiciones 
en las cuales están llevando a cabo la materialización de sus actividades no son las más apropiadas. En consideración de lo expuesto, se deja por sentado que en actividades como la mencionada no se cumplen requisitos tales como el pago de la seguridad social, de un salario justo, cesantías, primas y demás emolumentos.

La población afro descendiente, ha estado sometida a los continuos y degradantes tratos inhumanos que en ejercicio de las funciones propias de un cargo o empleo debe ejercer, todo ello dado que este tipo de población es discriminada, no solo por su color de pie, sino además por, sus creencias religiosas, culturales y por supuesto por su condición de analfabetismo. La cotidianidad nos demuestra que en la mayoría de los casos, la población negra de nuestro país, está trabajando en condiciones que a la luz de la constitución política de Colombia, va en contra vía del tan luminoso estado social de derecho, no solo porque es increíble ver como aun con una constitución tan relativamente perfecta, se siga presentando este tipo de situaciones, donde la mayoría de la población del país, específicamente los afro, se ven obligados a llevar una vida llena de necesidades, tropiezos y barreras que impiden un avance en el transcurrir normal de su vida. Por supuesto que el estado social de derecho se ha quedado corto frente a este tipo de temas y problemáticas, y más aún cuando en nuestro país el $60 \%$ de los recursos públicos son ejecutados de manera irregular, de manera indebida, o se les da un manejo inapropiado, concluyendo con esto que la mayoría de la población vulnerable de Colombia sigue en situación denigrante porque a los recursos públicos se les da un manejo que solo está favoreciendo a una clase privilegiada, dejando de lado el sentido único de la carta magna y del Estado Social de derecho.

\section{Derecho a la etnoeducación}

Los grupos Afrocolombianos a lo largo de su historia han consolidado una rica y fructífera cultura. Como no sentirse orgulloso de su lengua palenquera, de su exótica gastronomía, por sus danzas típicas y por sus matices religiosos. La Corte Constitucional se refirió así a la cultura Raizal que aunque con matices propios sí guarda una estrecha relación con los Afrocolombianos: "La cultura de las personas raizales de las Islas es diferente de la cultura del resto de los colombianos, 
particularmente en materia de lengua, religión y costumbres, que le confieren al raizal una cierta identidad. Tal diversidad es reconocida y protegida por el Estado y tiene la calidad de riqueza de la Nación. El incremento de la emigración hacia las Islas, tanto por parte de colombianos no residentes como de extranjeros, ha venido atentando contra la identidad cultural de los raizales, en la medida en que por ejemplo en San Andrés ellos no son ya la población mayoritaria, viéndose así comprometida la conservación del patrimonio cultural nativo, que es también patrimonio de toda la Nación." (Corte Constitucional, 1993).

En la Constitución Política, el concepto de etnoeducación es considerado como uno de los principios y derechos fundamentales de los individuos y de la sociedad. Varios artículos se refieren a la diversidad étnica y cultural:

a) El reconocimiento y proyección de la diversidad étnica y cultural de la nación colombiana (Artículo $7^{\circ}$ ).

b) Oficialización de las lenguas y dialectos de los grupos étnicos en sus respectivos territorios y obligatoriedad de una enseñanza bilingüe (Artículo 10).

c) La consideración de la cultura en sus distintas manifestaciones como fundamento de la nacionalidad y la necesidad de su investigación, desarrollo y divulgación (Artículo 70 Superior).

d) El Artículo 68 Superior establece que los integrantes de los grupos étnicos tendrán derecho a una formación que respete y desarrolle su identidad.

e) El Artículo 55 transitorio trata sobre "protección de la identidad cultural y derechos de las comunidades negras". La Ley 70 de 1993 reglamenta el Artículo aludido, sobre la protección de la identidad cultural y derechos de las Comunidades Negras, para ello crea la Cátedra de Estudios Afrocolombianos. (Ministerio de Educación Nacional, 2001)

La etnoeducación, por tanto, es el medio más idóneo para lograr que el Afrocolombiano interiorice y reconozca su identidad como parte de su existencia y consolide el camino que lo conducirá al fortalecimiento de su riqueza cultural. La 
Ley de Educación (Ley 115 de 1994) consagra en el artículo 56 que la educación a los grupos étnicos debe estar orientada por los principios y fines generales de la educación establecidos en la misma disposición normativa y tener en cuenta además los criterios de integralidad, interculturalidad, diversidad lingüística, participación comunitaria, flexibilidad y progresividad. Toda actividad educativa dirigida a esta población debe tener como finalidad afianzar sus procesos de identidad, socialización, protección y uso adecuado del medio ambiente, prácticas comunitarias de organización, uso de las lenguas vernáculas, formación docente e investigación en todos los ámbitos de la cultura.

A pesar de que el artículo 36 de la Ley 70 de 1993 es claro en determinar que "El Estado velará para que en el sistema nacional educativo se conozca y se difunda el conocimiento de las prácticas culturales propias de las comunidades negras y sus aportes a la historia y a la cultura colombiana, a fin de que ofrezcan una información equitativa $y$ formativa de las sociedades y culturas de estas comunidades. En las áreas de sociales de los diferentes niveles educativos se incluirá la cátedra de estudios afrocolombianos conforme con los currículos correspondientes.", tal deber y obligación no está siendo cumplida a cabalidad por algunas de las instituciones educativas visitadas, lo cual muestra una vez más, que somos un país que se conforma con el poder simbólico de las normas.

Es lamentable que los Afrocolombianos de Neiva, Baraya y La Plata no tengan la posibilidad de acceder a una educación étnica pertinente, y peor aún, que la cátedra de estudios Afrocolombianos no se esté impartiendo en debida forma.

\section{Derecho a la consulta previa}

El convenio 169 de la O.I.T., ratificado por Colombia mediante la Ley 21 de 1991, reconoció el derecho de los pueblos indígenas y tribales a ser consultados, "cada vez que se prevean medidas legislativas o administrativas susceptibles de afectarles directamente". 
Así, el derecho de participación de las comunidades étnicas y el derecho a la consulta previa derivada del convenio 169 , son vinculantes para el estado por las siguientes razones:

-"Porque el artículo $6^{\circ}$ del Convenio 169, aprobado por la Ley 21 de 1991, dispone que los pueblos indígenas y tribales tienen derecho a ser consultados, previamente, sobre las decisiones y medidas administrativas y legislativas que los afecten, sin restricciones.

-Dado que ninguna de las disposiciones del Convenio puede dar lugar a la asimilación de los pueblos indígenas y tribales a la cultura mayoritaria.

- Debido a que los derechos y deberes consagrados en el ordenamiento constitucional se interpretan de conformidad con los tratados internacionales sobre derechos humanos ratificados por Colombia."

-En razón de que el Convenio 169 de la OIT es un instrumento del derecho internacional de los derechos humanos, y el de mayor significación en el respeto de los derechos de las minorías étnicas." (Corte Constitucional Colombiana, 2003)

La corte constitucional colombiana ha establecido que el mencionado convenio cobija también a las comunidades negras, puesto que "la norma internacional en comento hace referencia a dos requisitos que deben concurrir a la hora de establecer quiénes se pueden considerar como sus beneficiarios: (i) Un elemento "objetivo", a saber, la existencia de rasgos culturales y sociales compartidos por los miembros del grupo, que les diferencien de los demás sectores sociales, y (ii) un elemento "subjetivo", esto es, la existencia de una identidad grupal que lleve a los individuos a asumirse como miembros de la colectividad en cuestión. De la definición legal que consagra el artículo 2-5 de la Ley 70/93, se desprende que las comunidades negras cumplen con esta doble condición, y por ende se ubican bajo el supuesto normativo del Convenio mencionado" (Corte Constitucional, 201).

Así mismo, para la Corte es claro que "El fundamento teórico de la doctrina constitucional que estima que el derecho de consulta de las comunidades étnicas 
es de naturaleza fundamental, toma pie en el carácter pluriétnico y multicultural de la Nación colombiana reconocido y protegido por la Carta, así como en el sello participativo de nuestra democracia y en el derecho de las comunidades a autodeterminarse" (Corte Constitucional, 2010).

A pesar de existir claridad sobre el deber de consultar a las comunidades negras, en no pocas ocasiones el mismo Estado se ha encargado de desconocer este derecho fundamental. El ejecutivo y el legislativo han pretendido desoír estos claros mandatos, al tomar decisiones administrativas y legislativas sin consultar las justas exigencias de estas comunidades, o queriendo mostrar un aparente proceso de consulta. Ante estos casos preocupantes de violación de derechos fundamentales, las comunidades se han visto obligadas a acudir a la justicia para reclamar por vía de tutela y demandas de inconstitucionalidad el respeto del derecho a la consulta previa.

\section{ANÁLISIS DE RESULTADOS}

\section{Los afrocolombianos en Neiva}

En Neiva se realizaron 45 entrevistas a hombres y mujeres blancas (Hombres: 18 y Mujeres: 27); de las personas encuestadas, relación con los Hombres Blancos tenemos: en promedio de edad: Entre 18 a 30 años: 8 y Mayores de 30 años 10 hombres. Casados: 5, Solteros: 10 y en Unión Libre: 3. Procedentes de los barrios Cambulos, Centro, Santander, Batallón Tenerife, Cándido, San Carlos, Siete de Agosto, Las Palmas I y II, Guillermo Lievano, Ventilador, Alberto Galindo, José Eustacio Rivera, Chíchala, Orquídea y el Jardín. Sobre Escolaridad se encuestaron 8 son estudios de secundaria, 1 tecnólogo y 9 universitarios. La mayoría de mujeres y hombres blancos no tendrían problema alguno en trabajar con un Afro, es más, manifiestan no tener ningún reparo de compartir con ellos una actividad social, pero cuando se trata de entablar relaciones afectivas o sentimentales las opiniones son distintas. 
1. ¿le gustaría tener como compañero (a) de trabajo a un negro

(a)?

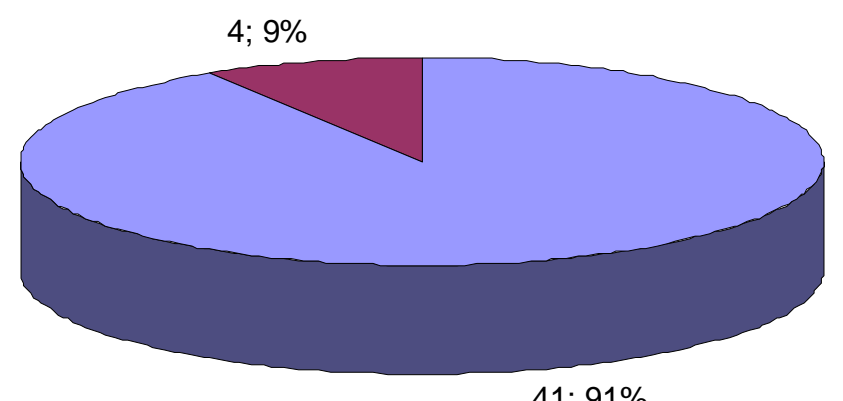

Mientras que el $91 \%$ de los Encuestados (41 Personas) no les afecta trabajar con Afrocolombianos, cuando se trata de tener relaciones sentimentales el $56 \%$ niega toda posibilidad de enamorarse de un Afro, aunque un $44 \%$ no le afectaría tener como pareja a una persona negra.

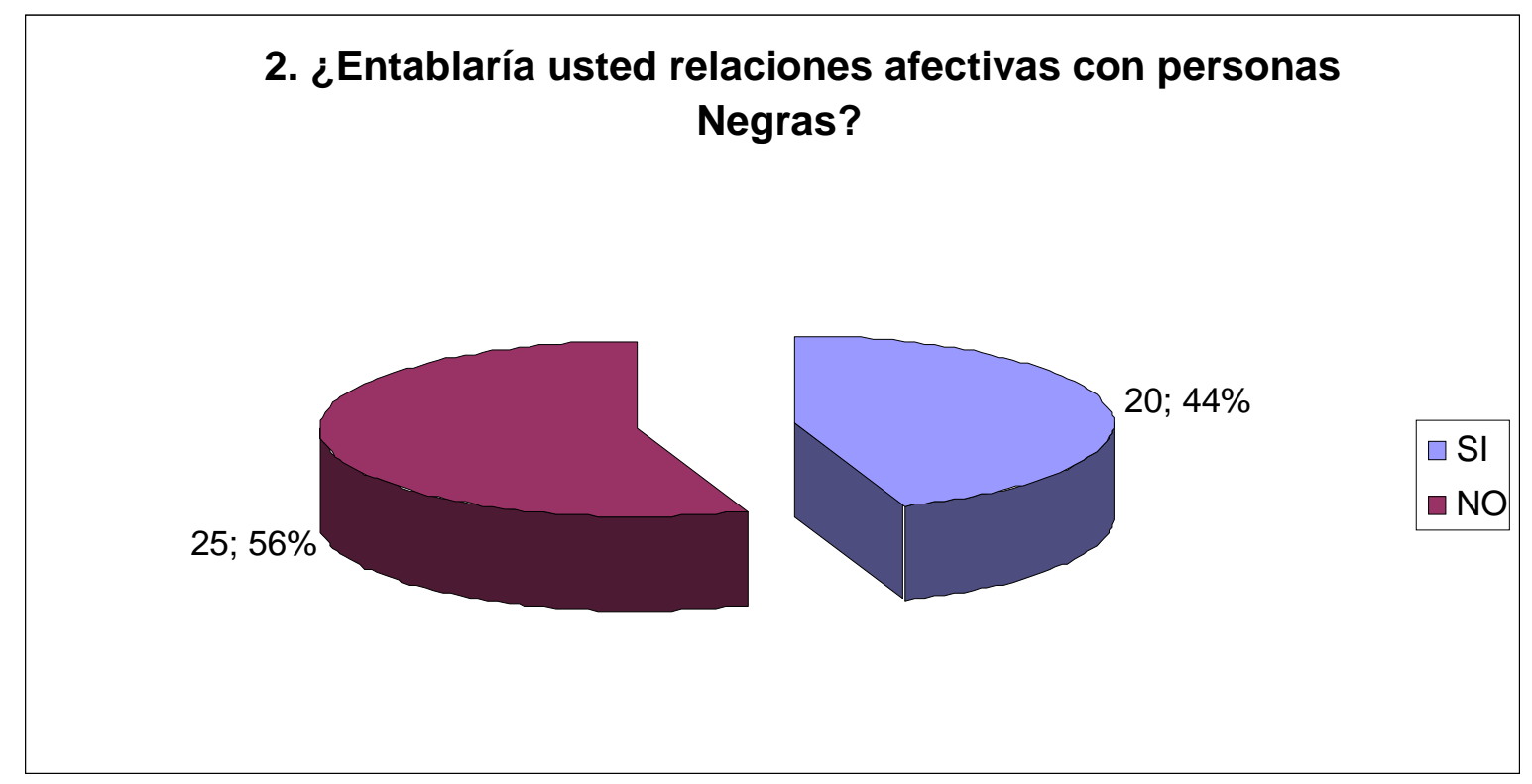

Lamentablemente comprobamos que existe en Neiva discriminación e indiferencia frente estas conductas excluyentes: 


\section{3. ¿Ha tenido conocimiento de casos de discriminación social (Vecinos, grupos sociales, en la calle) a negros?}

$28 ; 62 \%$

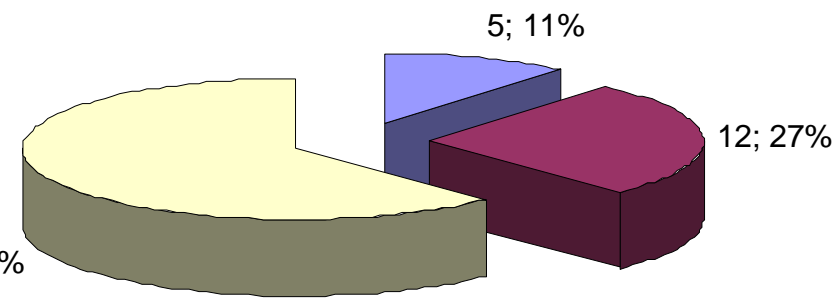

$\square$ SIEMPRE

$\square$ NUNCA

$\square$ ALGUNAS VECES

El $62 \%$ de los encuestados han observados alguna vez en su vida un hecho de discriminación y un $11 \%$ manifiesta haber sido siempre testigo de tan aberrantes conductas. Reconocen que la discriminación se presenta generalmente de manera verbal y en algunos casos psicológicos, aunque han sabido de situaciones donde los afrocolombianos han sido víctimas de maltrato físico.

\section{4. ¿Cómo se actúa frente a la discriminación?}

$12 ; 36 \%$

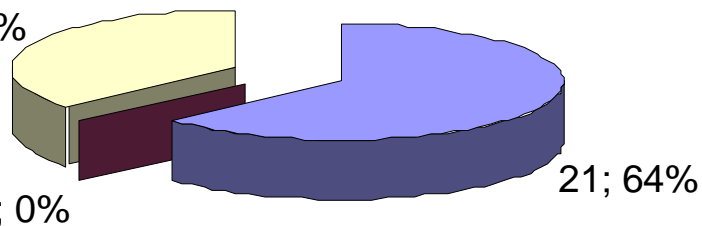

$0 ; 0 \%$ $\square$ INDIFERENCIA

AGRESIÓN FISICA

AGRESION VERBAL

El $64 \%$ de las personas blancas aceptaron actuar con indiferencia ante las conductas discriminatorias y excluyentes de los neivanos contra los Afro, solo un $36 \%$ comentó su rechazo a tales prácticas agrediendo verbalmente a las personas que discriminan.

Sin embargo, es menester resaltar que las personas blancas reconocen que los Afrocolombianos son personas capaces y muy inteligentes, especialmente como docentes, deportistas y artistas. 
Además de dialogar con personas blancas, fue posible encuestar directamente a los Afrocolombianos en procura de identificar, desde su perspectiva, si consideran - no que la población neivana es sensible y reconoce sus Derechos Fundamentales. Para ello se realizaron 34 Entrevistas: 18 Hombres y 16 Mujeres. En relación con los Hombres Negros se estableció: En promedio de edad: Menores de 18 años: 1, Entre 18 a 30 años: 10 y Mayores de 30 años: 7. Casados: 4, Solteros: 10 y en Unión Libre: 4. Procedentes de los barrios Cambulos, Palmas, Kennedy, Minuto de Dios, Tenerife, Santa Inés, Alberto Galindo, Chíchala, José Eustacio Rivera, Santander y Ventilador. Sobre la Escolaridad: 3 con estudios de Primaria, 8 con Secundaría, 5 Tecnólogos y 2 Universitarios. Respecto de la Actividad Laboral: tenemos 5 Comerciantes, 3 Independientes, 2 Maestros de Construcción, 2 en las Fuerzas Militares, 1 Docente y 2 desempleados. En materia de Seguridad Social: 12 vinculados al Sistema y 6 no vinculados.

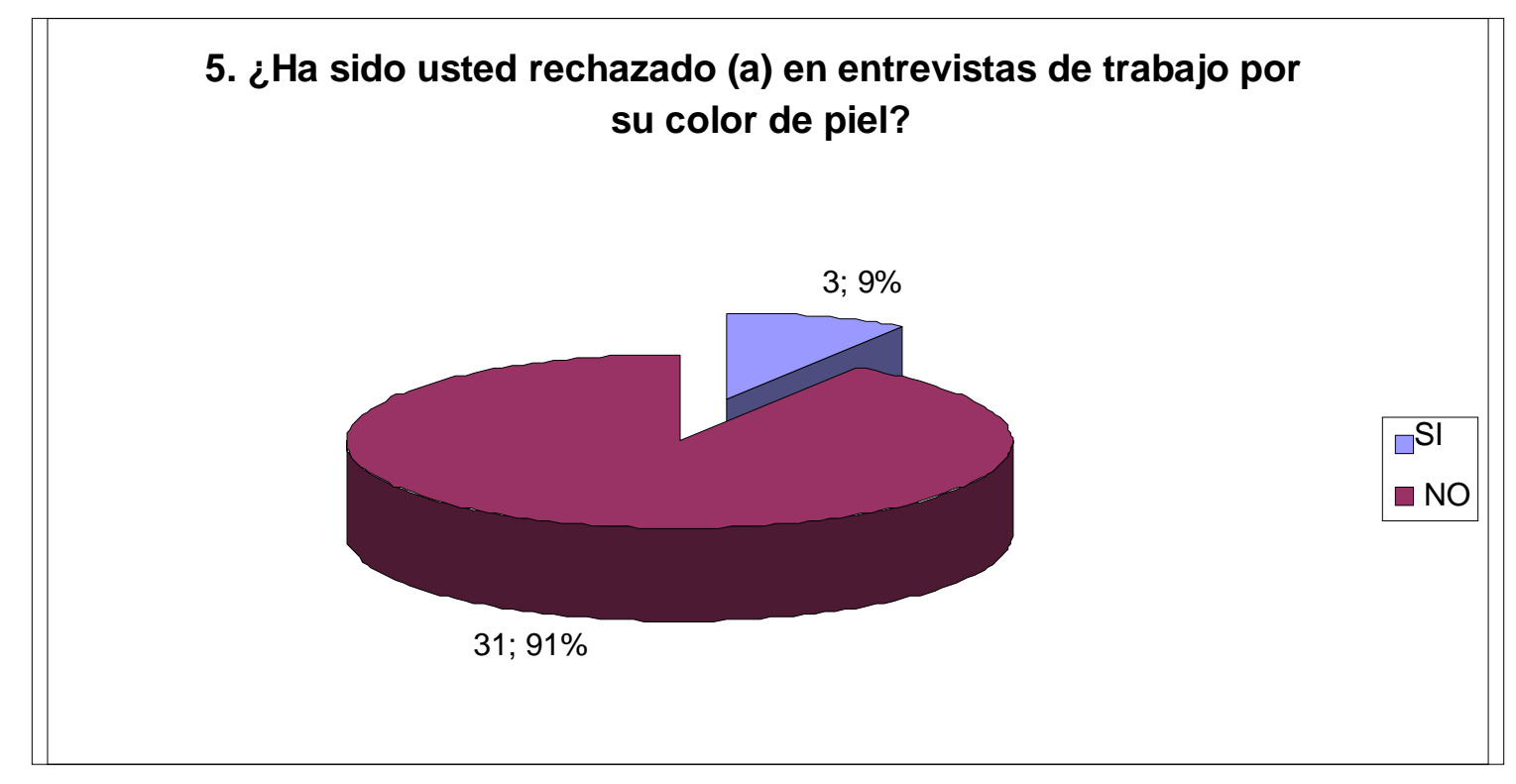

Aunque sus respuestas coinciden con las posturas de las personas blancas sobre la no discriminación radical en materia laboral algunos se sienten excluidos. El $91 \%$ no ha sido rechazado en una entrevista de trabajo por su color, pero aun un $9 \%$ de los encuestados han sido víctima de estas posturas de intolerancia. 


\section{6. ¿Le han prohibido la entrada en algún sitio público por su Color de piel?}

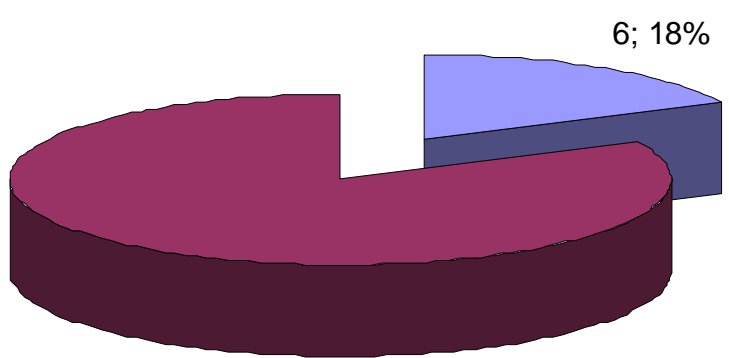

$28 ; 82 \%$

En lo que respecta a su vida social, si bien es cierto el $82 \%$ expresa no haber tenido la molestia de habérsele impedido la entrada aún lugar público, el 18\% si ha sufrido tan dolorosa experiencia.

\section{7. ¿Ha tenido dificultades al entablar relaciones afectivas con Personas blancas por el color de su piel?}

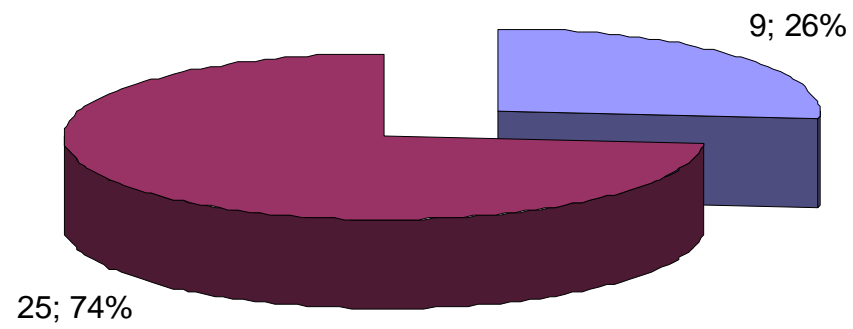

Todavía se evidencia un rechazo a estas personas de color negro pese a todo el discurso de equidad y de respeto a la dignidad humana, a la solidaridad y al reconocimiento de la diversidad cultural pues queda en el vació al momento de amar y de formar una familia. Aunque la mayoría afirmó no haber tenido dificultades en este tema, es pertinente que aun existen problemas: El $74 \%$ ha logrado encontrar en su vida a seres humanos sensibles que valoran a los demás por sus valores y principios, sin importar aspectos superfluos como el color de la piel, pero el $26 \%$ ha tenido dificultades al entablar una relación efectiva con una persona blanca. 
Ahora bien, además de acercarnos a blancos y a Afrocolombianos resolvimos abordar algunas Instituciones Educativas públicas y privadas: Gimnasio Yumaná, Cooperativo Salesiano, Santa Clara de Hungría, Ateneo Autónomo de Colombia, Rafael Pombo, Piayet, Mundo de los Niños, El Bosque, INEM, Normal Superior, San Miguel Arcángel, Misael Pastrana Borrero, Humberto Tafur, Ricardo Borrero Álvarez, para tener una visión aproximada sobre el impacto que tienen estas entidades en las comunidades Afro y en sus procesos de formación. Sobre el particular pudimos concluir que el número de estudiantes Afro matriculados en los Centros Educativos de Neiva, es notoriamente bajo con relación a la cantidad de alumnos blancos o de otras etnias. Asimismo ocurre en cuanto a los empleados administrativos y docentes de las instituciones, el número empleados afro es reducido en lo que respecta a otros grupos poblacionales; No obstante, hay que hacer mención de que los pocos Negros que laboran en el sector de la educación, trabajan como profesores de Educación Física.

\section{8. ¿Conoce de experiencias de discriminación hacia los Afrocolombianos que estudian o laboral en las Instituciones encuestadas?}

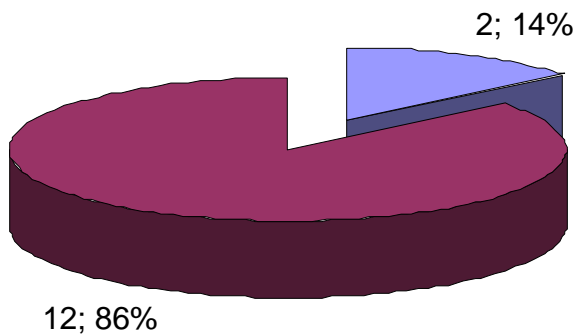

Nuestros niños, niñas y jóvenes afrocolombianos, aunque en un porcentaje relativamente bajo (14\%), aun son víctimas de discriminación racial en las Instituciones Educativas de la ciudad de Neiva. Corresponden a las familias neivanas y a los colegios de primaria y básica secundaria formar a sus hijos y estudiantes en el respeto a la diferencia y al reconocimiento a la diversidad cultural, a la dignidad humana y a la solidaridad.

Si bien es cierto, según así lo indicaron el $86 \%$ de los docentes encuestados, no hay discriminación hacia los Afrocolombianos que laboran o estudian en las Instituciones educativos, no es suficiente. Debe ser compromiso de todos erradicar de la vida social cualquier práctica aberrante que atente contra los seres humanos por su color o diferencia. 


\section{9. ¿Se desarrolla en las Instituciones la Cátedra de "Estudios Afrocolombianos", en el Área de Ciencias Sociales, conforme lo ordena la Ley 70 de 1.993 y el Decreto 1122 de $1.998 ?$}
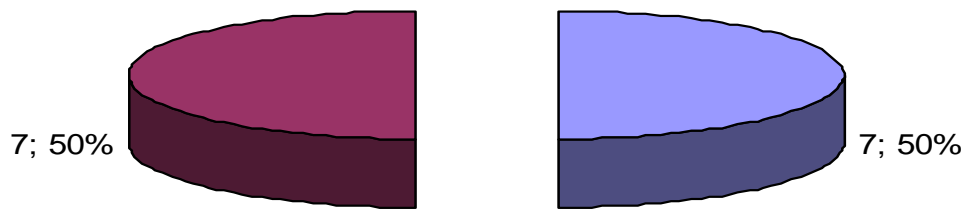

La investigación dentro del aula de clase conlleva la ineludible conclusión de que las instituciones no tienen políticas especiales referentes el sistema de ingreso, permanencia y promoción para estas minorías étnicas, además de que tienen muy poco conocimiento sobre la fecha en que se celebra el día de la Afrocolombianidad a nivel nacional establecido en ley 725 de 2001, y que tan solo la mitad de Instituciones Educativas desarrollan la cátedra de estudios Afrocolombianos en el área de Ciencias Sociales, conforme así lo consagra la Ley 70 de 1993 y el Decreto 1122 de 1998.

\section{Los afrocolombianos en Baraya}

De entrada, en materia de Educación, se infiere que la situación de los afros en Baraya es alarmante, dado que si bien este municipio cuenta con un gran número de instituciones educativas, no hay implementación alguna de la cátedra de Afrocolombianidad. Además de lo anterior, se llega a la concepción de que es muy preocupante la situación en la población afro, no solo desde el punto de vista de la no implementación de la cátedra, sino que la mayoría de la población negra entrevistada, posee un nivel muy bajo de estudios, casi tocando el umbral del analfabetismo.

Las siguientes gráficas ilustran los resultados arrojados en la Entidad Territorial acerca de la situación de la población afro descendiente en materia de educación. 


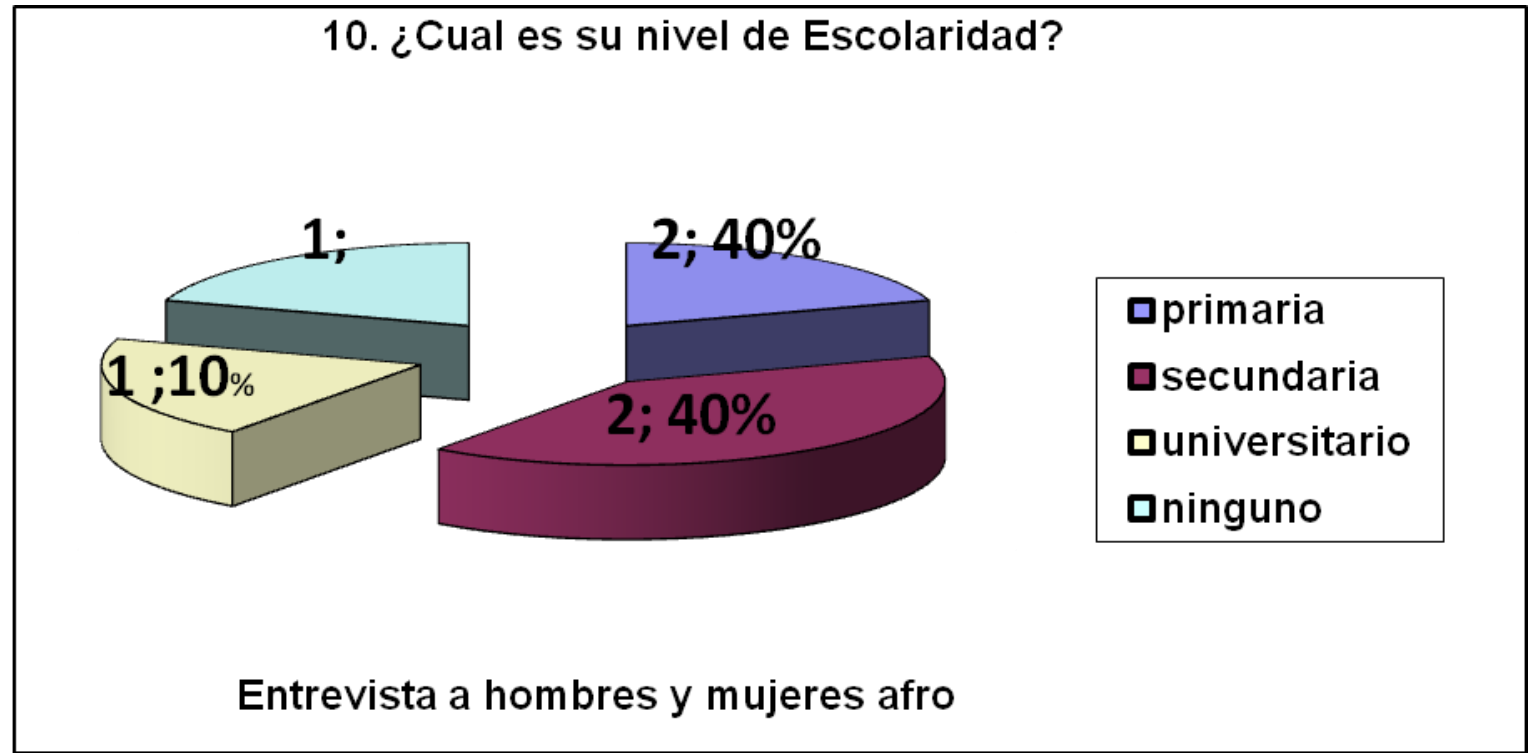

Teniendo en cuenta que la población afro descendiente es estadísticamente baja, dado que la dependencia encargada de llevar el registro y censo del número de personas que pertenecen a este tipo de población en el municipio, no tiene información alguna sobre las personas afrocolombianas que habitan en dicha región, se procedió a aplicar este método de investigación a 6 personas afro habitantes del lugar, arrojando los siguientes resultados:

De las seis personas entrevistadas, una de ellas expresó no tener ningún tipo de contacto con la academia, dado que en tiempos de juventud no tuvo las oportunidades económicas y familiares para poder asistir a una escuela con el fin poder obtener una formación educativa digna. Tres afirmaron haber realizado estudios de educación media, uno de ellos concluyendo satisfactoriamente su bachillerato. Sin embargo de los encuestados, solo fue posible encontrar una persona con formación académica superior, quien se desempeña como docente en el mismo municipio y una persona que solo ha realizado estudios de primaria hasta el tercer grado.

Frente a la implementación de la cátedra de derechos humanos, en ninguna de las instituciones educativas del municipio de Baraya, se ha venido dando desarrollo, implementación, publicidad, ni mucho menos impulso programático de la mencionada cátedra, que por supuesto de mucha importancia, teniendo en cuenta que es a través de esta, donde los estudiantes recopilan información y demás 
conocimientos frente a los temas en general que tienen que ver con la Afrocolombianidad.

\section{1. ¿Ha sido usted rechazado (a) en entrevistas de trabajo por su color de piel?}

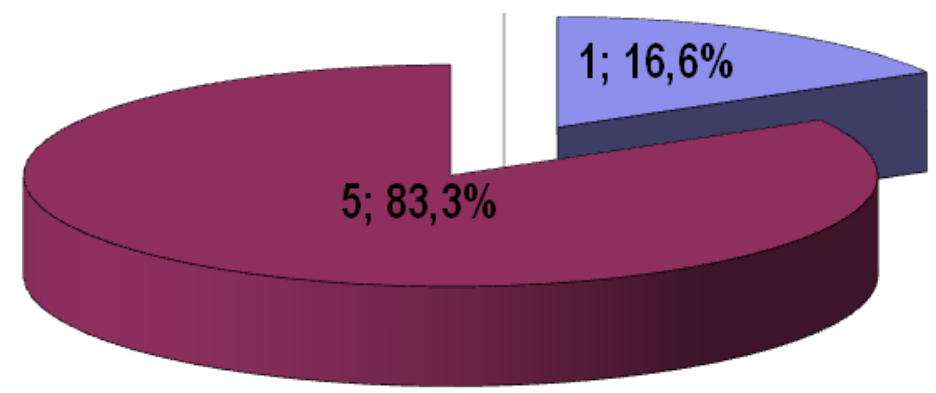

$\square$ SI $\square$ NO

Al realizarse la pregunta ¿Ha sido usted rechazado (a) en entrevistas de trabajo por el color de su piel?, 5 personas afro descendientes de las 6 encuestadas, manifestaron no haber recibido ningún tipo de rechazo por parte de las personas que realizan las entrevistas al momento de aplicar a alguna vacante o empleo en dicho municipio, además se pudo establecer que al momento de querer ingresar a algún trabajo, estas personas son tratadas con decoro, respeto y sobre todo sin recibir discriminación alguna por parte de las personas encargadas de seleccionar el personal.

Sin embargo una de las personas encuestadas, que en el grafico anterior representa el $16.6 \%$, manifestó que en varias ocasiones, ha sido objeto de discriminación y rechazo, al momento de querer aplicar a una entrevista laboral, con el fin de poder obtener el empleo ofertado.

Por lo visto, en el municipio de Baraya, aunque es muy poca la población afro descendiente, se puede inferir, que la discriminación por motivos de color es baja. Casi siempre, quien contrata o para quien trabajan, son personas de la misma zona, por lo cual la mayoría de las veces se deja de lado la realización de entrevistas, para evaluar las condiciones de la persona que desea laborar, debido a que casi siempre se trabaja en el campo, desarrollando actividades propias de la agricultura, entonces allí se omite dicha formalidad al momento de realizar la contratación. 


\section{Los afrocolombianos en la Plata}

A continuación se estima pertinente detallar algunas conclusiones obtenidas con ocasión al municipio de la Plata sobre el ejercicio y respecto de los derechos fundamentales de los Afrocolombianos en la localidad.

\section{Grafica 12.}

¿Ha sido usted rechazado (a) en entrevistas de trabajo por su color de piel?

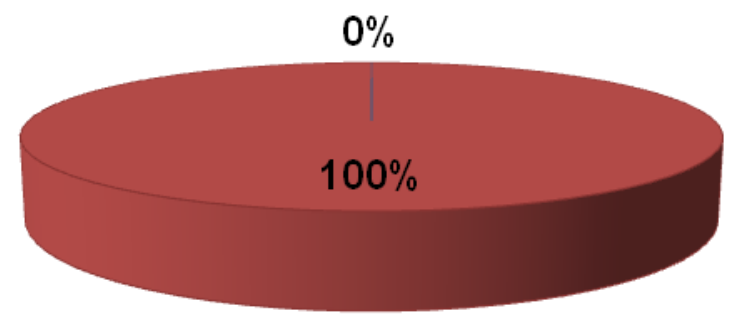

$\square \mathrm{SI} \quad \square \mathrm{NO}$

La comunidad afro descendiente oriunda o residente en el municipio de la Plata (Huila), considera que no ha sido discriminada, por su color de piel, cuando ha requerido un trabajo, sin embargo los encuestados no dudaron en afirmar que sienten temor a rechazo al momento de la entrevista laboral. Es decir, si bien es cierto se podría concluir que la Plata registra un importante avance en el proceso de atenuación y eliminación de la discriminación hacia los afro descendientes, aun persiste, en ellos, la sensación de exclusión y marginación.

La constante se repite, los encuestados manifestaron no haber tenido problemas a la hora de entablar relaciones afectivas con personas blancas. Al parecer en este municipio la discriminación de los blancos hacia los negros no existe, permitiendo que los afros tengan una mejor integración con la sociedad y su entorno.

Como parte del trabajo de investigación fue medir la discriminación en materia afectiva, se hizo esta pregunta a personas no afrocolombianas. Los resultados demuestran que aún persiste la discriminación en materia afectiva de las personas blancas hacia los negros, más es valorable que el $97 \%$ de los encuestados rechacen esta práctica y estén dispuestos a prodigar afecto a las personas afro descendientes. 


\section{CONCLUSIONES}

En relación con la difícil y compleja relación de la sociedad colombiana con sus Afrocolombianos son válidas las afirmaciones del Doctor Juan de Dios Mosquera, reconocido líder de esta etnia en el país:

El racismo o prejuicio racial en Colombia, es el conjunto de ideas y fijaciones sicológicas de discriminación racial mantenidas en el inconsciente colectivo de nuestra sociedad. Es una herencia ideológica de la colonia esclavista, recreada y reproducida por nuestros días por la penetración cultural de los estados unidos (Mosquera, J. 2007)

La situación de abandono y discriminación del pueblo afro es lamentable: "En el año 2003 , el $47,2 \%$ de esta población no tenía sus necesidades básicas satisfechas y vivían en condiciones de extrema pobreza. El ingreso salarial promedio de los afrocolombianos es aproximadamente $20 \%$ menor al del promedio de las personas no afro y tan solo el $51 \%$ pueden acceder al sistema de seguridad social en salud. La desventaja de la población afrocolombiana en el acceso a la salud resulta evidente cuando se considera que el $53,5 \%$ de los hogares afrocolombianos pobres en las cabeceras municipales y el $73,8 \%$ de los rurales carecen de un régimen de salud. La esperanza de vida También es muy inferior al promedio nacional que, de los 65 años de vida, pasa a los 55 para los afrocolombianos. Lo mismo sucede con la nutrición: el $14 \%$ de los afrocolombianos pasaron por lo menos un día entero sin comer en la semana del censo de 2005, es decir, más de dos veces la cifra, también preocupante, de los mestizos que aguantan hambre. Los niveles de educación entre los habitantes afrocolombianos también son inferiores a los de la población mestiza: mientras que el $12,4 \%$ de los hombres afrocolombianos terminaron la educación superior, el $18,5 \%$ de los hombres mestizos lo hicieron. A su vez el 13,5\% de las mujeres afrocolombianas terminaron la educación superior frente al 19,7\% de mujeres mestizas que lo hicieron. La tasa de analfabetismo de hombres y mujeres afro son superiores a las de los hombres y mujeres mestizos: el 18,4\% de hombres y el $16,9 \%$ de mujeres afro son analfabetas, mientras que el $13,2 \%$ de hombres y el 
$11,7 \%$ de mujeres mestizas lo son. Los niños afrocolombianos tienen menos tiempo para estudiar, pues trabajan más que los niños mestizos: $2,56 \%$ de los afrocolombianos menores de 12 años que trabajaron en la semana anterior al censo de 2005, a su vez el 0,79\% de los mestizos menores de 12 años lo hizo. El total de niños que trabajaron en la semana anterior al censo es de 1,63\%" (Observatorio de Discriminación Racial).

Colombia se ha visto marcada por episodios de violencia, destrucción y de violaciones flagrantes a los derechos constitucionales y fundamentales de cada una de las personas que habitan dentro del territorio, derivado ello de los continuos problemas de orden público, social y económico que aquejan nuestro país, desde hace ya más de 60 años. Es continuo observar, como día tras día en el cotidiano transcurrir de la vida, los índices de violencia y de desarticulación social, han llegado a cobrar muchas víctimas, más de las que el país se ha acostumbrado a vivenciar. Colombia es un país que en la actualidad, se encuentra sumergido en una gran crisis social y económica, y es aquí donde se hace aguda la relación de cada uno de los habitantes del territorio, pues las continuas agresiones contra la vida, la integridad y demás derechos y bienes jurídicos tutelables son el pan de cada día.

La discriminación por razón de raza, sexo y color es una de las problemáticas que aquejan nuestra sociedad en el avanzado estado de modernidad en el cual nos encontramos. Un ejemplo claro de ello, son los famosos cabeza rapadas quienes partiendo de sus objetivos ideológicos han propiciado lamentables hechos de discriminación y violencia hacia algunos sectores de la sociedad. Así mismo es constante ver discriminación frente a los grupos indígenas, ciertos sectores que profesan un tipo de cultura diferente de la de las personas del común, discriminación hacia grupos religiosos, políticos y sobre todo frente a grupos afro descendientes.

En Colombia actualmente, se observan elevados índices de discriminación por razón del color de las personas, generando síntomas de descontento por parte de grupos sociales que han salido a la defensa de este tipo de población vulnerada 
con el fin de hacer garantizar y hacer respetar los derechos de estas personas, que por el solo hecho de tener un color de piel diferente, vienen siendo tratados como un "espécimen o vicho raro" a las personas del común. Organizaciones como el movimiento nacional CIMARRON en cabeza del Doctor Juan de Dios Mosquera Mosquera, el movimiento nacional Afrocolombiano, la dependencia de Asuntos para Comunidades Negras, Afrocolombianas, Raizales y Palenqueras del Ministerio del Interior y de Justicia, además de las diferentes asociaciones $u$ organizaciones que están establecidos dentro de cada departamento y municipio como por ejemplo la asociación de Afrocolombianos del Huila.

Al momento de aplicar, las encuestas a personas del común de Neiva, Baraya y la Plata acerca de si tenían conocimiento alguno sobre casos de discriminación, hacia personas de color, el $66.6 \%$ de los encuestados respondieron afirmativamente, estableciendo que han tenido conocimiento de actos discriminatorios de diversa índole, como discriminación traducida en agresiones verbales, físicas y psicológicas por parte de personas de color blanco, que de una u otra forma, han demostrado tener un alto grado de repudio hacia personas de color de piel negra, ya sea por su aspecto físico, cultural, ideológico o económico.

El restante $33.3 \%$ de los encuestados, manifestaron no tener ningún tipo de conocimiento frente a actos discriminatorios encaminados a afectar a personas Afrocolombianas con motivo de su raza, sexo o color. Por el contrario han establecido estas personas, que la mayoría de las veces en las cuales han tenido contacto con personas afro descendientes, han sustraído de los mismos una muy buena relación social y así mismo las personas que son más próximas a estas no han manifestado señales de agresión a este tipio de personas. Sin embargo han realizado algún tipo de reparo, en los cuales se han pronunciado al respecto y han hecho un llamado de atención a toda la población de los diferentes lugares del país, para que no marginemos a estas personas que son tan iguales como lo somos cada uno de nosotros respecto de los demás.

Un acto de discriminación racial lesiona no sólo los derechos singulares de una persona. En muchos casos, puede también comprometer los derechos de la 
comunidad o de una etnia. (Corte Constitucional, 1996). En palabras de la honorable corte constitucional, se tiene que todas aquellas conductas que están viabilizadas a ejercer un daño o atentado de los derechos fundamentales de una determinada persona por motivo de su raza o color, además de vulnerar particularmente cada uno de sus derechos, así como de los bienes jurídicos protegibles, se atenta contra el interés general y los derechos de una colectividad cual es la etnia o comunidad a la cual pertenece esa persona, que en el caso particular sería un Afrocolombiano. Es decir que al momento de ejercer actos discriminatorios sobre una persona de color, no solo se estaría atentando contra sus derechos, sino que además se estarían vulnerando los derechos de toda la comunidad Afrocolombiana asentada en el país y fuera de él, todo ello teniendo en cuenta que la comunidad negra integra un todo, dado que es uno de los tipos de población "especiales" reconocidos legalmente por el ordenamiento jurídico colombiano. En otras palabras, todo lo anterior significa, que al momento de ejercer conductas discriminatorias sobre una persona "negra" negra en particular, se está atentando además contra la comunidad afro a la cual pertenece.

Por último, hay que resaltar que las diferentes organizaciones de comunidades afro descendientes en casi la totalidad en el mundo, han realizado una estupenda labor frente a la protección de esta comunidad especial, dado que estos han adoptado medidas tendientes a garantizar y defender los intereses de dichas poblaciones, frente a los tratamientos discriminatorios por parte de terceros.

Además hay que observar la gran importancia, que en el tema ha adquirido cada uno de los países que han desarrollado cantidad de instrumentos de derecho internacional e interno, con los cuales se pretende proteger las comunidades negras, de toda conducta orientada a vulnerar los derechos fundamentales de estos, así como el proteger de cualquier atentado contra los bienes jurídicos que en cabeza de estos deben garantizarse. A partir de lo anterior, se rescata aquí un instrumento de gran valor cuya utilización es de vital significación frente al continuo accionar tendiente a eliminar todo tipo de acto discriminatorio a personas 
de color, como es la Convención Internacional sobre la Eliminación de todas las Formas de Discriminación Racial.

En el Departamento del Huila, concretamente en su ciudad capital y en los Municipios de Baraya y la Plata aunque la situación no es complicada como en otras regiones del país, no puede dejarse de lado que aún hay vestigios de conductas discriminatorias contra la población Afrocolombiana que conlleva a la amenazada o a la violación de sus Derechos Fundamentales afectando su calidad de vida personal, social y familiar.

Es necesario que tanto el Departamento como los municipios del Huila se comprometan con sus gentes, promoviendo e implementando en las Instituciones Educativas la Cátedra de Afrocolombianidad como lo ordena la Ley, así como también impulse actividades pedagógicas dirigidas a rescatar y consolidar las costumbres de esta etnia como base fundamental en la consolidación de un Estado Social de Derecho Democrático y Plurietnico.

\section{REFERENCIAS BIBLIOGRÁFÍCAS}

- colombia, ministerio dE EdUCACIÓN NACIONAL (2001). Lineamientos curriculares Cátedra Estudios Afrocolombianos.

- CASTILLO, L. (2006). El Estado-Nación pluriétnico y multicultural colombiano: la lucha por el territorio en la reimaginación de la nación y la reivindicación de la identidad étnica de negros e indígenas. Madrid.

- colombia, DEPARTAMENTO ADMINISTRATIVO NACIONAL DE ESTADÍSTICA (2010).

- COlOMBia, CORTE COSNTItUCIONAL, "Sentencia T - 422 de 1996", M.P. Cifuentes Muñoz, E.

- COlOMBiA, CORTE COSNTITUCIONAL, "Sentencia C - 169 de 2001", M.P. Gaviria Díaz, C. 
- COlOMBIA, CORTE COSNTITUCIONAL, "Sentencia C - 702 de 2010", M.P. Pretelt Chaljub, J.I.

- COlOMBia, CORTE COSNTItUCIONAL, "Sentencia C - 530 de 1993", M.P. Martínez Caballero, A.

- COlOMBia, CORTE COSNTITUCIONAL, "Sentencia SU - 383 de 2003", M.P. Tafur Galvis, A.

- MOSQUERA, J. (2007). La población afrocolombiana: realidad, derechos y organización (Quinta edición). Bogotá: Movimiento Nacional por los Derechos Humanos de las Comunidades Afrocolombianas.

- OBSERVATORIO DE DISCRIMINACIÓN RACIAL. Población Afrocolombiana, Discriminación Racial y Derechos Humanos [on line]. Disponible en:

http://www.ddhhcolombia.org.co/files/Cifras\%20sobre\%20discriminacion\%20racial. pdf.

- URREA, F. y BARBAY, O. (2004). Gente negra en Colombia. Bogotá: Lealon. 